\title{
Occam Paradox? A Variation of Tapia Syndrome and an Unreported Complication of Guidewire- Assisted Pedicle Screw Insertion
}

\author{
Osa Emohare ${ }^{1,2}$ Erik Peterson ${ }^{1} \quad$ Nathaniel Slinkard $^{3}$ \\ ${ }^{1}$ Center for Spine and Spinal Cord Injury, Regions Hospital, St. Paul, \\ Minnesota, United States \\ 2 Department of Orthopaedic Surgery, University of Minnesota, \\ Minneapolis, Minnesota, United States \\ ${ }^{3}$ Department of Orthopaedic Surgery, Henry Ford Health System, \\ Detroit, Michigan, United States \\ ${ }^{4}$ Department of Otolaryngology, Regions Hospital, St. Paul, \\ Minnesota, United States \\ ${ }^{5}$ Department of Otolaryngology, University of Minnesota, \\ Minneapolis, Minnesota, United States
}

\author{
Seth Janus ${ }^{4,5}$ Robert Morgan ${ }^{1,2}$
}

Address for correspondence Osa Emohare, MBBS, PhD, Center for Spine and Spinal Cord Injury, Regions Hospital, 640 Jackson Street, St. Paul, MN 55101, United States (e-mail: oemohare@umn.edu).

Evid Based Spine Care J 2013;4:132-136.

\begin{abstract}
Study Design Case report.

Clinical Question The clinical aim is to report on a previously unknown association between guidewire-assisted pedicle screw insertion and neuropraxia of the recurrent laryngeal nerve (RLN), and how this may overlap with the signs of Tapia syndrome; we also report our approach to the clinical management of this patient.

Methods A 17-year-old male patient with idiopathic scoliosis experienced Tapia syndrome after posterior instrumentation and arthrodesis at the level of T1-L1. After extubation, the patient had a hoarse voice and difficulty in swallowing. Imaging showed a breach in the cortex of the anterior body of T1 corresponding to the RLN on the right. Results Otolaryngological examination noted right vocal fold immobility, decreased sensation of the endolarynx, and pooling of secretions on flexible laryngoscopy that indicated right-sided cranial nerve $X$ injury and left-sided tongue deviation. Aspiration during a modified barium swallow prompted insertion of a percutaneous endoscopic gastrostomy tube before the patient was sent home. On postoperative day 20 , a barium swallow demonstrated reduced aspiration, and the patient reported complete resolu-

\section{Keywords}

- pedicle screw

- cortical breach

- guidewire

- tapia syndrome

- neuropraxia tion of symptoms. The feeding tube was removed, and the patient resumed a normal diet 1 month later. Tapia syndrome, or persistent unilateral laryngeal and hypoglossal paralysis, is an uncommon neuropraxia, which has previously not been observed in association with a breached vertebral body at T1 along the course of the RLN.

Conclusion Tapia syndrome should be a differential diagnostic consideration whenever these symptoms persist postoperatively and spine surgeons should be aware of this as a potential complication of guidewires in spinal instrumentation.
\end{abstract}

received

February 21, 2013

accepted

July 18,2013 (c) 2013 Georg Thieme Verlag KG

Stuttgart - New York
DOI http://dx.doi.org/ 10.1055/s-0033-1357355. ISSN 1663-7976. 


\section{Introduction}

Postoperative neuropraxia of the lower cranial nerves ( $\mathrm{CNs}$ ) can occur as a complication of spinal surgery, manifesting as a hoarse voice, impaired breathing, and tongue paresis. Although these features are common immediately after extubation, their persistence has been termed Tapia syndrome. There have been isolated reports of bilateral cases, but Tapia syndrome is a unilateral disorder with ipsilateral paresis of the tongue and vocal cord. ${ }^{1-3}$ The general consensus from the literature is that the syndrome results from a pressure neuropathy or traction of local structures during procedures such as tracheostomy, intubation, or thoracotomy. ${ }^{4-10}$ The use of guidewires during pedicle screw insertion has not been associated with this syndrome. We present a patient who experienced persistent difficulty breathing and dysphagia after surgery. Subsequent review of postoperative imaging was normal, apart from a breach in the cortex of the anterior body of the T1 vertebra that corresponded anatomically to the path of the recurrent laryngeal nerve (RLN) on the right. ${ }^{11}$

\section{Case Report}

A 17-year-old male patient with a history of idiopathic scoliosis underwent a posterior instrumentation and arthrodesis at T1-L1. General anesthesia was induced with sevoflurane and propofol. An 8.0 endotracheal tube was placed uneventfully, and the patient was positioned prone on a Jackson table. The surgery lasted for 10 hours and 35 minutes with no intraoperative complications, and the patient was kept intubated because of the length of the procedure and the time spent in the prone position. After extubation on the first postoperative day (POD), the patient had a hoarse voice and difficulty in swallowing (including coughing when attempting to swallow liquids). Extensive neurological imaging, including magnetic resonance image (MRI) of the brain and the brain stem, magnetic resonance angiograph of the neck, and MRI of the cervical spine was performed but no abnormalities were found (-Fig. 1). Computed tomography (CT) demonstrated a small breach of the cortex at the level of the T1 vertebra (-Fig. 2).

An otolaryngology review noted right vocal fold immobility, decreased sensation of the endolarynx, and pooling of secretions on flexible laryngoscopy that indicated a rightsided CN X injury and a left-sided tongue deviation which is suggestive of a left-sided CN XII injury. Aspiration was noted during a modified barium swallow, prompting insertion of a percutaneous endoscopic gastrostomy (PEG) tube. In addition, augmentation of the true vocal cord was performed by injecting hyaluronic acid. The patient left the hospital on POD 9 on PEG tube feeding. On POD 20, a repeat of the barium swallow demonstrated a reduced level of aspiration, and an examination showed complete resolution of symptoms. The PEG tube was removed, and the patient returned to a normal diet 1 month later.

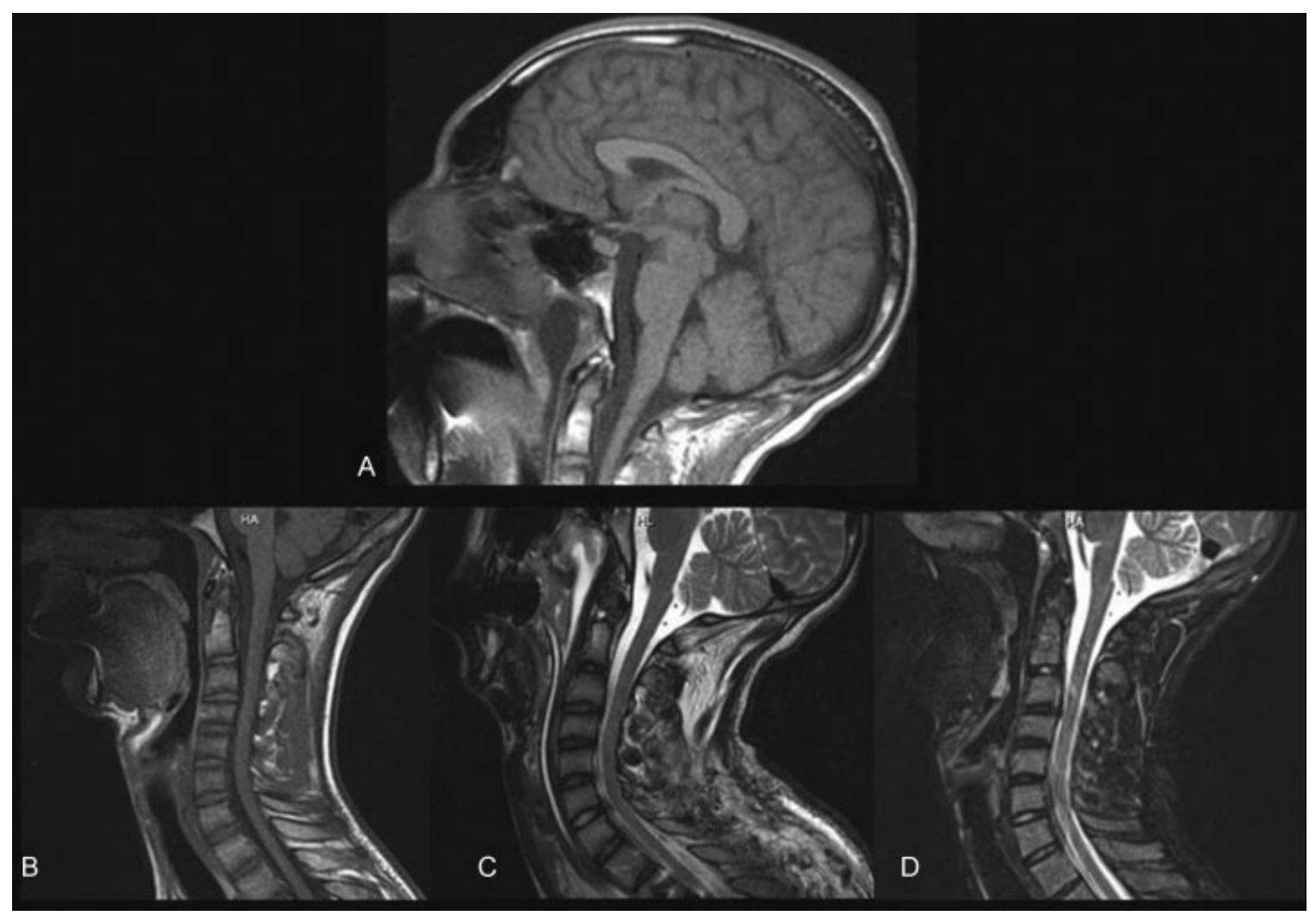

Fig. 1 (A) T1 Magnetic resonance image (MRI) of the brain. (B) T1, (C) T2, and (D) STIR sequence MRI of the cervical spine taken after the patient's symptoms started, showing no clear evidence of injury. 


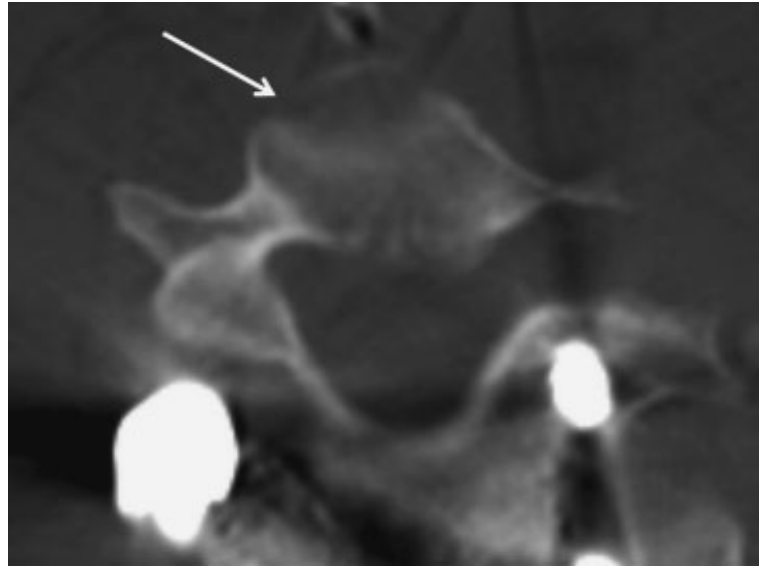

Fig. 2 Computed tomography of the thorax at the level of T1 with a breach of the cortex of the body of T1 on the right side.

\section{Discussion}

Tapia syndrome is a posttraumatic or postsurgical ipsilateral neuropraxia that can be unilateral ${ }^{1,6-9,12}$ or bilateral. ${ }^{4,13}$ Its pathophysiologic factors are not completely understood. One theory is that positioning the patient with the neck in anterior and lateral flexion can impinge both $\mathrm{CN} X$ and $\mathrm{CN}$ XII where the two nerves cross. ${ }^{4,6,7}$ Cinar et $\mathrm{al}^{4}$ reported on bilateral Tapia syndrome following rhinoplasty, and although they stated that there was no clear etiology, they attributed the nerve injury to changing positions of the neck, malposition of the endotracheal tube, compression by the endotracheal tube, or a combination of these. Boisseau et $\mathrm{al}^{6}$ described a case of Tapia syndrome after arthroscopic shoulder surgery. In this case, marked lateral flexion may have stretched the CNs for a prolonged time or placed increased pressure around the orotracheal tube. Other pathophysiologic theories include overinflated endotracheal tube cuffs, ${ }^{8}$ malpositioned endotracheal tubes, ${ }^{6,7}$ carotid or vertebral artery dissection ${ }^{12,14}$ aneurysm, ${ }^{15}$ and increased pressure on the lateral roots of the tongue by the tongue blade during intubation. ${ }^{7,9,15}$ All published studies report resolution of symptoms within 2 years. ${ }^{4,6-9,16}$

To understand the pathophysiologic factors of Tapia syndrome, an appreciation of the function and anatomic relationships of CN XII and CN X in the neck is required. After exiting the base of the skull through the hypoglossal canal, CN XII travels in the carotid sheath before exiting at roughly the $\mathrm{C} 2$ level and traveling anteriorly ${ }^{9,17}$ ( $\mathbf{- F i g . 3 )}$. CN XII then travels superior to the hyoid bone to terminate and provide motor innervations to the intrinsic muscles of the tongue. $\mathrm{CN} \mathrm{X}$ descends in the carotid sheath and branches into the superior laryngeal nerve, which then divides into the external laryngeal nerve, providing motor innervation to the cricothyroid muscle. It then branches into the internal laryngeal nerve, providing sensory innervations of the larynx to the level of the true vocal folds. The RLN consists of the terminal branches of CN X after coursing around the aortic arch on the left and

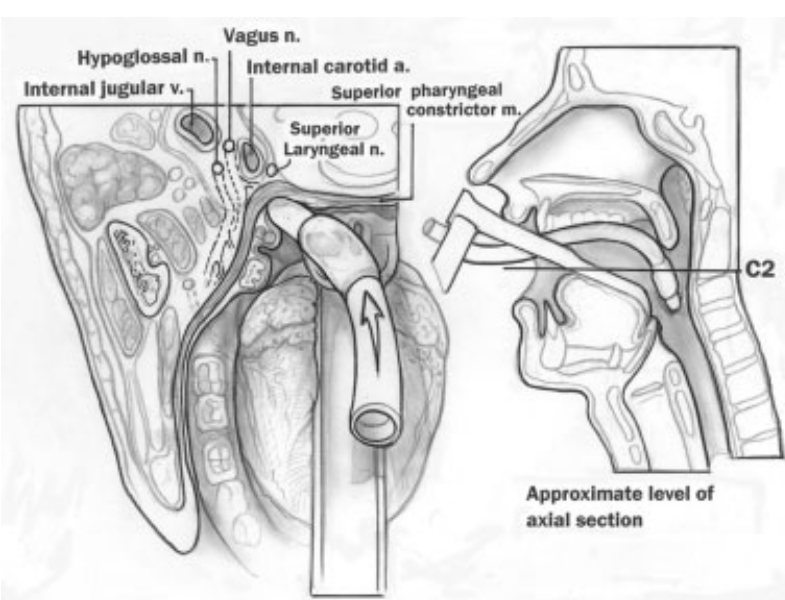

Fig. 3 Artistic illustration of an axial section of the head and neck at the approximate level of C2. It shows the hypoglossal and vagus nerves as they begin to travel along an anterior course and out of the carotid sheath to their final innervation structures.

the subclavian on the right. The RLN ascends the neck in the tracheoesophageal groove and terminates at the level of the larynx, where it provides motor innervation to the remaining intrinsic muscles of the larynx as well as sensory innervation of the larynx inferior to the true vocal folds and the trachea

(-Fig. 4).

In the case presented, the etiology of the patient's symptoms seems to be the cortical breach in the body of the T1 vertebra (-Fig. 2). Recent anatomic dissections demonstrate the proximity of the path of the RLN to the T1 vertebra, especially on the right side. ${ }^{11}$ The presence of concomitant

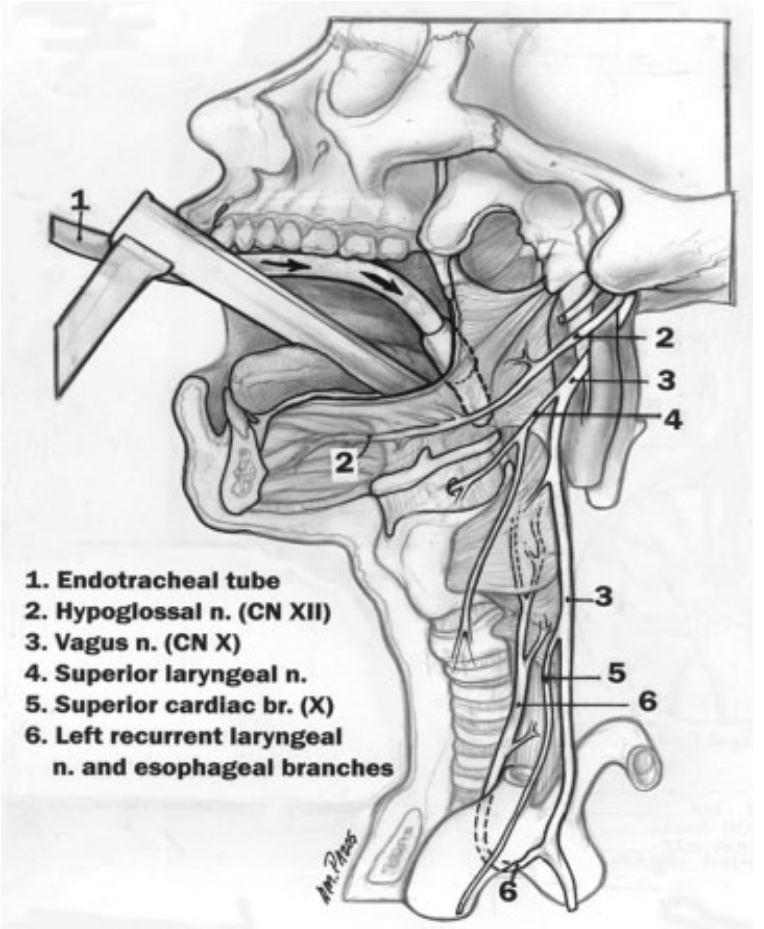

Fig. 4 Artistic illustration showing the path of the endotracheal tube during intubation and the sites where nerve injury could occur. 
impairments in opposing CN X (right sided) and CN XII (left sided) is intriguing because Tapia syndrome is normally unilateral $^{1}$; when bilateral cases have been described, ${ }^{4,13}$ the neuropathies have been ipsilateral and contiguous. This underlying principle may be explained by the close proximity of CN X and CN XII, and in $10 \%$ of one cadaveric series, the inferior ganglion of the vagus nerve was bound to the trunk of the hypoglossal nerve. ${ }^{18}$

According to some researchers, Tapia syndrome is caused by stretching of the nerves, use of endotracheal or orotracheal cuffs, positioning of the patient, or direct trauma to the area. Regardless of the etiology, the one common theme is trauma to the upper cervical soft tissues. Surgeons should pay particular attention to the course of the RLN, especially on the right side, in patients undergoing spinal instrumentation. Although it occurs very infrequently, the possibility of Tapia syndrome should inform management and decision making in procedures involving the upper airways, especially when symptoms of dysphonia, dysphagia, and deviation of the tongue occur immediately after surgery or extubation. Treatment is mainly supportive and aimed at minimizing the effect of the neuropraxia. With early recognition and appropriate support, more severe complications can be avoided.

\section{Acknowledgments}

We thank Mary Van Beusekom for her assistance in the preparation and review of this manuscript.

\section{Disclosure}

This report was exempt from institutional review board's review; no external support was received for this project.

\section{Device status}

The device was FDA approved.

\section{References}

1 Tapia AG. Un caso de paralisis del lado derecho de la laringe y de la ungue, con paralisis del externo-cleidomastoidea y trapecio del mismo lado. Siglo Medica 1905;52:211-213

2 Schoenberg BS, Massey EW. Tapia's syndrome. The erratic evolution of an eponym. Arch Neurol 1979;36(5):257-260

3 Haymaker W. Bing's Local Diagnosis in Neurological Diseases. 15th ed. St. Louis, MO: CV Mosby Co; 1969:170-185
4 Cinar SO, Seven H, Cinar U, Turgut S. Isolated bilateral paralysis of the hypoglossal and recurrent laryngeal nerves (Bilateral Tapia's syndrome) after transoral intubation for general anesthesia. Acta Anaesthesiol Scand 2005;49(1):98-99

5 Park J, Ahn R, Weon Y, Yang D. Diagnosing Tapia Syndrome Using a Videofluoroscopic Swallowing Study and Electromyography After Anterior Cervical Spine Surgery. Am J Phys Med Rehabil 2011; 90:948-953

6 Boisseau N, Rabarijaona H, Grimaud D, Raucoules-Aimé M. Tapia's syndrome following shoulder surgery. Br J Anaesth 2002;88(6): 869-870

7 Tesei F, Poveda LM, Strali W, Tosi L, Magnani G, Farneti G. Unilateral laryngeal and hypoglossal paralysis (Tapia's syndrome) following rhinoplasty in general anaesthesia: case report and review of the literature. Acta Otorhinolaryngol Ital 2006;26(4): 219-221

8 Kashyap SA, Patterson AR, Loukota RA, Kelly G. Tapia's syndrome after repair of a fractured mandible. Br J Oral Maxillofac Surg 2010; 48(1):53-54

9 Gelmers HJ. Tapia's syndrome after thoracotomy. Arch Otolaryngol 1983;109(9):622-623

10 Rotondo F, De Paulis S, Modoni A, Schiavello R. Peripheral Tapia's syndrome after cardiac surgery. Eur J Anaesthesiol 2010;27(6): 575-576

11 Haller JM, Iwanik M, Shen FH. Clinically relevant anatomy of recurrent laryngeal nerve. Spine(Phila Pa 1976) 2012;37(2): 97-100

12 Johnson TM, Moore HJ. Cranial nerve X and XII paralysis (Tapia's syndrome) after an interscalene brachial plexus block for a left shoulder Mumford procedure. Anesthesiology 1999;90(1): 311-312

13 Turan I, Yildirim ZK, Tan H. Bilateral Tapia syndrome secondary to oropharyngeal intubation. J Neurosurg Anesthesiol 2012;24(1):78

14 Al-Sihan M Jr, Schumacher M, Löhle E. Tapia syndrome caused by a vertebral artery dissection. Ear Nose Throat J 2011;90(7):313314

15 Shimohata T, Nakano R, Sato S, Tsuji S. [A patient with aneurysm of extracranial internal carotid artery presenting lower cranial polyneuropathy similar to Tapia's syndrome]. Rinsho Shinkeigaku 1994;34(7):707-711

16 Yavuzer R, Başterzi Y, Ozköse Z, Yücel Demir H, Yilmaz M, Ceylan A. Tapia's syndrome following septorhinoplasty. Aesthetic Plast Surg 2004;28(4):208-211

17 Alves P. Imaging the hypoglossal nerve. Eur J Radiol 2010;74(2): 368-377

18 Saraswathi P. Communication between the vagus and hypoglossal nerves. Eur J Anat 2003;7(3):131-134

\section{Commentary}

\author{
Carlos Bagley ${ }^{1}$ \\ ${ }^{1}$ Department of Neurosurgery, Duke University Medical Center, \\ Durham, North Carolina, United States
}

The authors present an interesting case of a patient who underwent a multilevel fusion for spinal deformity using guidewire-assisted pedicle screw fixation that was found to have a variant of Tapia syndrome postoperatively. The literature regarding this rare syndrome has been reported following various surgical procedures and is due to injury of cranial nerves (CNs) X and XII. ${ }^{1}$ Although the exact etiology of the $\mathrm{CN}$ dysfunction is not completely clear in this case, it 
is illustrative of the various complications that we may encounter in spinal surgery as techniques continue to evolve. ${ }^{2,3}$ Various guidewire-related complications have been reported in the literature; however, this report of an associated cranial nerve palsy is an unusual, yet important, potential complication that those of us in the spine community should be cognizant. Fortunately, as was the case for this patient, the overall prognosis for recovery remains excellent, with most cases resolving completely within 6 months. ${ }^{1}$ As we continue to push the proverbial envelope, we must continue to weigh the potential risk/ benefit ratio of all that we do, keeping in mind to first do no harm. The authors are to be applauded for their very interesting report and their critical analysis of this patient's postoperative findings in diagnosing this rare clinical syndrome.

\section{References}

1 Lykoudis EG, Seretis K. Tapia's syndrome: an unexpected but real complication of rhinoplasty: case report and literature review. Aesthetic Plast Surg 2012;36(3):557-559

2 Wang MY, Block S. Retrieval of retained Jamshidi needle fragments during minimally invasive surgery. J Neurosurg Spine 2011;14(5): 681-684

3 Wang MY, Mummaneni PV. Minimally invasive surgery for thoracolumbar spinal deformity: initial clinical experience with clinical and radiographic outcomes. Neurosurg Focus 2010;28(3):E9

\section{Editorial Perspective}

EBSJ thanks the authors for offering an honest review of an unusual case and for expanding our knowledge base by describing Tapia syndrome. Although a case report, this is a good application for this form of scientific contribution due to its rarity. Dr. Bagley's helpful discussion underscores the danger of minimally invasive procedures, which usually rely on some form of cannulation or threading maneuver, and which may result in unexpected secondary findings. 